\title{
Correlation Between Promoter Hypomethy
and Increased Expression of Syncytin-I in Non-Small Cell Lung Cancer
}

This article was published in the following Dove Press journal: International Journal of General Medicine

\author{
Yang $\mathrm{Fu}^{\prime}$ \\ Xuewei Zhuang $\mathbb{D}^{2,3}$ \\ Xiyan $\mathrm{Xia}^{4}$ \\ Xiaohui $\mathrm{Li}^{3}$ \\ $\mathrm{Ke} \mathrm{Xiao}^{3}$ \\ Xiaojing $\mathrm{Liu}^{3}$ \\ 'Department of Reproductive Medicine \\ Center, Jinan Maternity and Child Care \\ Hospital, Jinan, 25000I, People's Republic \\ of China; ${ }^{2}$ Department of Clinical \\ Laboratory Medicine, Shandong \\ Provincial Third Hospital, Cheeloo \\ College of Medicine, Shandong \\ University, Jinan, 25003I, People's \\ Republic of China; ${ }^{3}$ Qilu Hospital, \\ Cheeloo College of Medicine, Shandong \\ University, Jinan, 2500 I2, People's \\ Republic of China; ${ }^{4}$ Department of \\ Microbial Immune, Jinan Vocational \\ College of Nursing, Jinan, 2500 I2, \\ People's Republic of China
}

Introduction: Syncytin-1 is a human endogenous retroviral (HERVW) envelope protein, which has been implicated in trophoblast and cancer cell fusions as well as in immunomodulatory functions. We investigated syncytin-1 expression and promoter methylation in non-small cell lung cancer (NSCLC) and the adjacent, para-carcinoma tissues. In addition, the correlation to patient survival differentiation of between 5-year survival and death group was analyzed.

Methods: Survival ratio was calculated by Kaplan-Meier survival curve. Death risk assessment was executed by Cox risk regression model. The 5'-LTR methylation level of HERVW promoter was detected by EpiTYPER method.

Results: Syncytin-1 expression in NSCLC tissue was found to be significantly higher than in para-carcinoma tissues. Moreover, the 5-year survival group has a lower syncytin-1 expression than the death group. Clinical stage and the percentage of syncytin-1 positive cells were top risk factors according to Cox ratio risk regression model analysis. While the methylation level of the 5'-LTR in HERVW gene promoter was relatively lower in NSCLC than paracarcinoma tissues, the methylation status of a CpG-2 site overlapping the Oct-1 binding site was found to be an important element potentially involved in the epigenetic regulation of $H E R V W$ gene expression.

Conclusion: These findings suggest that syncytin-1 could be a biomarker for the diagnosis/ prognosis of NSCLC, and further studies are required to elucidate the exact role of syncytin1 in the development of NSCLC as well as the underlying molecular mechanism for syncytin-1 function and regulation.

Keywords: non-small cell lung cancer, syncytin-1, epigenetic regulation, prognosis, DNA methylation

\section{Introduction}

More than $25 \%$ tumor mortality is caused by lung cancer worldwide, ${ }^{1}$ and $85 \%$ of lung cancers belong to non-small cell lung cancer. ${ }^{2}$ According to an updated report from the American Cancer Society (ACS), it was estimated that in 2020, 1,806,590 new cancer cases and 606,520 cancer deaths were projected to occur in the United States alone. ${ }^{3}$ Approximately $75 \%$ of lung cancers are diagnosed in late stages that are associated with a low 5-year survival rate of $15.6 \%{ }^{4,5}$ In these late stage cases, treatments including surgery, radiotherapy or chemotherapy are of very limited efficacy, highlighting an urgent need for early diagnosis and treatment.

Syncytin-1 is a functional glycoprotein encoded by the env gene located at chromosome 7q21-22. As a member of the human endogenous reverse transcription
Correspondence: Xuewei Zhuang

Tel +86-53I-8I656669

Fax +86-53I-86927544

Email zhuangxuewei@sdu.edu.cn 
virus $\mathrm{W}$ gene family, ${ }^{6,7}$ syncytin-1 is highly expressed in human placenta and several carcinoma tissues. Maliniemi et al. reported syncytin-1 expression in 15 of 30 epidermic malignant lymphoma. ${ }^{8}$ Sun et al. reported significantly increased expressions of syncytin- 1 in archaeocytes, lymphocytes, granulocytes and monocytes during acute myelocytic leukemia. ${ }^{9}$ Larsson et al. reported that $38 \%$ of 165 premenopausal node-negative breast cancers are positive for syncytin-1 expression, and this positivity correlates with the poor outcomes of patients. ${ }^{10}$ Strissel et al. disclosed that syncytin-1 is over-expressed in late-stage and poorly differentiated endometrial carcinoma rather than the early-stage and highly differentiated one. ${ }^{11}$ The expression of syncytin-1 in non-small cell lung cancer tissues remained poorly understood.

It is well recognized that genetic changes such as site mutation, deletion, translocation, and/or amplification as well as epigenetic changes including alterations in histone modifications, patterns of DNA methylation and/or microRNA can all contribute to the carcinogenesis of lung cancers. ${ }^{1,12}$ Multiple genetic mutations including translocation of $A L K$ gene, mutation of $E G F R$ gene and rearrangement of ROS-1 gene have been identified and the oncogenic mechanisms well characterized. ${ }^{13}$ Epigenetic changes, through their impact on gene transcription, may lead to activation of oncogene and silence of tumor suppressor genes. ${ }^{14}$ $H E R V W$ gene is not only overexpressed in various types of tumors, recent studies indicated that the gene products may be involved in modulation of cell cycle and cell apoptosis in placental trophoblasts. ${ }^{15}$ Its expression and epigenetic regulation in lung cancers, however, remains uninvestigated.

In this study, syncytin-1 expression in carcinoma and para-carcinoma tissues of NSCLC is detected by immunohistochemistry. The 5'-LTR methylation of HERVW promoter is determined by EpiTYPER method. Correlation of syncytin-1 expression and carcinogenesis of NSCLC is analyzed. These studies may provide novel information regarding its involvement in the carcinogenic mechanism of NSCLC. Such knowledge could be useful for the improvement of diagnosis, prognosis and management of NSCLC cases.

\section{Materials and Methods}

\section{Patients and Tissue Samples}

Thirty patients clinically diagnosed as NSCLC from Jan 1999 to Oct 2001 in Qilu Hospital, Shandong
University, were included in this study, of which 23 were male and 7 female, ages ranging from 37-75 (57.87 \pm 1.72 in average), 14 glandular and 16 squamous, 12 TNM stage I, 6 stage II and 12 stage III, 15 with lymphatic metastasis and 15 without. None of the patients received radiotherapy or chemotherapy before surgery. The inclusive criterion was: Survived for more than 1 month after surgery and did not die of any cause other than lung cancer during the follow-up time of 1-78 month (34 on average). Tissue samples were collected from pulmonary primary lesions with pathological characteristics identified under microscope. This study was authorized and administrated by the Ethics Committee of Qilu Hospital, Shandong University. Paracarcinoma refers to the adjacent regions $1-2 \mathrm{~cm}$ from the edge of carcinoma.

\section{Immunohistochemistry}

Carcinoma and para-carcinoma tissues removed in surgery were fixed with $10 \%$ neutral formalin for $12-24 \mathrm{~h}$ and embedded in paraffin. Tissues were sectioned into slides in thickness of $6 \mu \mathrm{m}$, dewaxed in xylene for $5 \mathrm{~min}$ twice, and dehydrated with $100 \%$, $95 \%, 85 \%, 75 \%$ alcohol for 60 seach. Tissue sections were boiled in EDTA repairing fluid for $2.5 \mathrm{~min}$ and rinsed with PBS three times for antigen retrieval after cooling. Endogenous peroxidase was blocked with $100 \mu \mathrm{L} 3 \%$ hydrogen peroxide by incubation at $37^{\circ} \mathrm{C}$ for $10 \mathrm{~min}$ before being rinsed with PBS three times. Antibody solution is obtained by 1:100 dilution from the rabbit anti-human syncytin-1 monoclonal antibody (GeneTex, USA). The tissue slides were exposed to $50 \mu \mathrm{L}$ of antibody solution at room temperature for $2 \mathrm{~h}$. After extensive rinsing with PBS, the tissue slides were stained with the secondary antibody (Zhongshan Jinqiao, Beijing) by incubation at $37^{\circ} \mathrm{C}$ for $30 \mathrm{~min}$. Color development was performed with $\mathrm{DAB}$ solution (Zhongshan Jinqiao, Beijing) for 3-5 min. After counter staining with hematoxylin, the slides were dehydrated, cleared, and mounted for microscopic observation.

\section{Evaluation of Staining}

Syncytin-1 protein in cytoplasm and cell membrane was positively stained in brownish color and the nuclei was in blue color. The percentage of positive cells was calculated, and semi-quantitative analysis was performed by categorization of the staining density into four classes: negative ( 0 positivity), weakly positive 
(lower than $25 \%$ positivity), moderately positive (25$50 \%$ positivity) and strongly positive (more than $50 \%$ positivity).

\section{EpiTYPER Methylation Detection}

The paraffin-fixed tissues were sliced into $10 \mu \mathrm{m}$ thickness, and 5-10 tissue slides were used for each DNA extraction. PCR primer sequences were designed by the EpiDesigner software: 5'-TTTTTTTTGGGATGAGGGTAAA and 3'TTAAAACAAACCCAAACACTTAACC. PCR amplification was executed at $94^{\circ} \mathrm{C}$ for $4 \mathrm{~min}$ as initial denaturalization, followed by 45 cycles of denaturalization at $94^{\circ} \mathrm{C}$ for $20 \mathrm{~s}$, annealing at $56^{\circ} \mathrm{C}$ for $30 \mathrm{~s}$, extension at $72^{\circ} \mathrm{C}$ for $1 \mathrm{~min}$, with hydrosulfite DNA as templates. The PCR products were visualized by $1.5 \%$ agarose gel electrophoresis under $160 \mathrm{~V}$ for $20 \mathrm{~min}$ as follows (Figure 1). PCR products were digested with SAP at $37^{\circ} \mathrm{C}$ for $20 \mathrm{~min}, 85^{\circ} \mathrm{C}$ for $5 \mathrm{~min}$, and dotted into MassArray sampler for mass spectroscopy detection. The results were analyzed with the EpiTYPER software.

\section{Statistical Analysis}

The syncytin-1 staining positivity of NSCLC carcinoma and para-carcinoma were compared with Student's t-test using the SPSS 17.0 statistical software, and $\mathrm{P}<0.05$ was considered statistically significant. Survival curve was constructed with the Kaplan-Meier method and intro-curve comparison was performed with the Log rank test. Prognosis was evaluated by Cox ratio risk recurrence model. The 5'-LTR methylation of $H E R V W$ promoter was analyzed by mass spectroscopy and EpiTYPER software. The average methylation ratio and standard deviation was calculated with the SPSS 17.0 statistical software, and compared with nonparametric rank sum test and Mann-Whitney $U$-test. $\mathrm{P}<0.05$ was considered statistically significant.

\section{Results}

Comparison of Syncytin-I Staining Positivity Between NSCLC Carcinoma and Para-Carcinoma Tissues

Syncytin-1 protein staining positivity was 96.7\% (29/30), with $65.5 \%(19 / 29)$ strongly positive (Figure 2A) and $34.5 \%$ (10/29) moderately positive (Figure 2B) in carcinoma tissues, and $33.3 \%$ lowly expressed in paracarcinoma tissues (Figure 2C). Syncytin-1 positive cell rate in carcinoma tissues $(35.41 \% \pm 0.22 \%)$ was significantly higher than in para-carcinoma tissues $(2.17 \%$ $\pm 0.03 \%)(\mathrm{P}<0.01)$ (Figure 3$)$. High expression is defined as higher than $25 \%$ positive cell rate.

\section{Comparison of Syncytin-I Expression Between 5-Year Survival and 5-Year Death Group}

Syncytin-1 expression positivity was $44.67 \% \pm 0.21 \%$ in the 5-year death group and $23.30 \% \pm 0.16 \%$ in the 5 -year survival group. The former was significantly higher than the latter $(\mathrm{P}<0.01)$ (Figure 4$)$.

\section{Survival Analysis}

Five-year mortality of syncytin-1 high-expression group was $73.7 \%$ and the low-expression group was $27.3 \%$, with a significant difference between the two groups $(\mathrm{P}<0.05)$. The average survival period of the low-expression group was 64 months while the highexpression group was only 28 months, and the difference was statistically significant $(\mathrm{P}<0.01) \quad$ (Figure 5) according to the Kaplan-Meier survival curve analysis. Clinical stage and positive cell percentage were found to be lethal factors of NSCLC based on the Cox ratio risk recurrence model (Table 1).

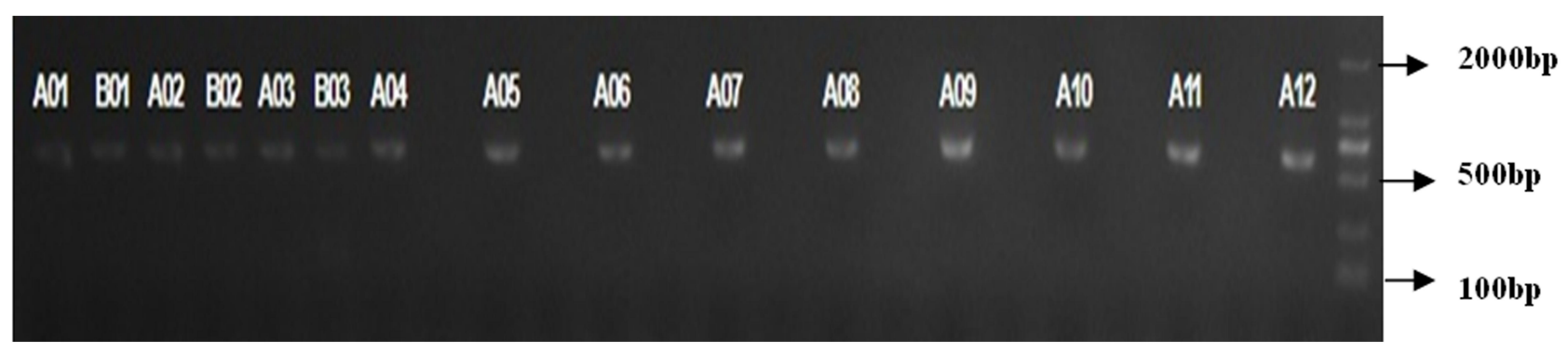

Figure I Detection for the PCR products of tissue DNA by I.5\% agarose gel electrophoresis. The marker: DL2000 bands are 2000 bp, 1000 bp, 750 bp, 500 bp, 250 bp, and 100 bp from top to bottom. 


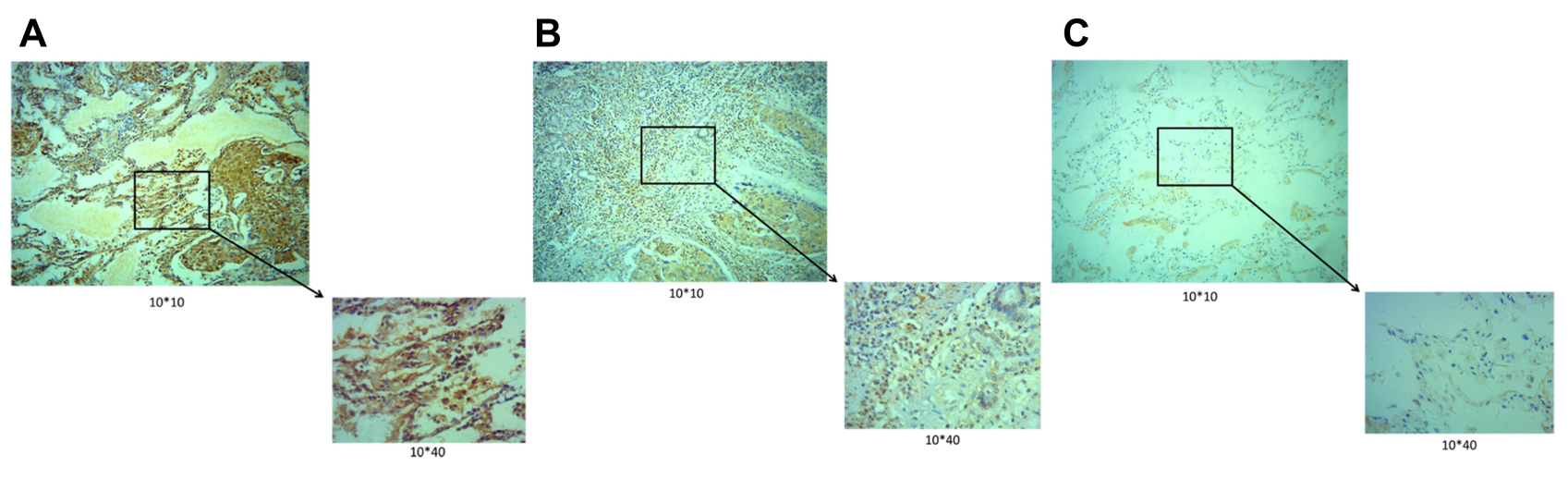

Figure 2 Syncytin-I staining in cancer tissue and para-cancerous tissue. Tumor cells were evident with strong syncytin-I staining (A), moderate syncytin-I staining (B) in cancer tissues and nearly no staining of syncytin-I in para-carcinoma tissues (C).

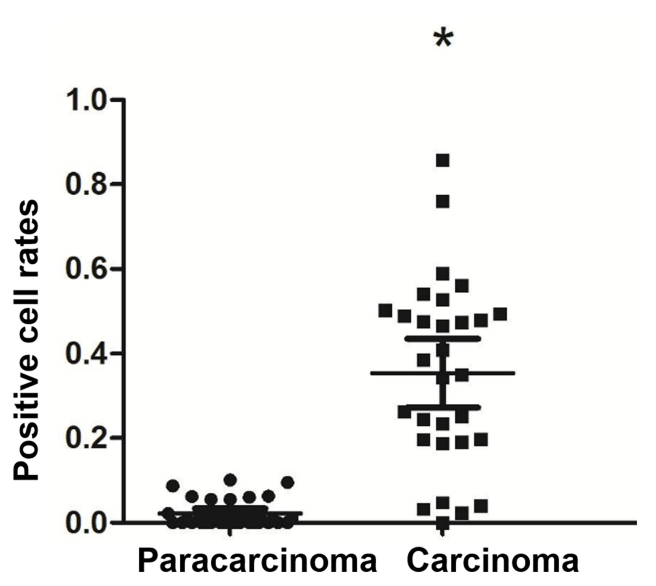

Figure 3 Syncytin-I positive cell rate in NSCLC tissues and para-carcinoma tissues. The syncytin-I positive cell rate in NSCLC tissues was higher than that in adjacent tissues. *Represents $\mathrm{P}<0.05$.

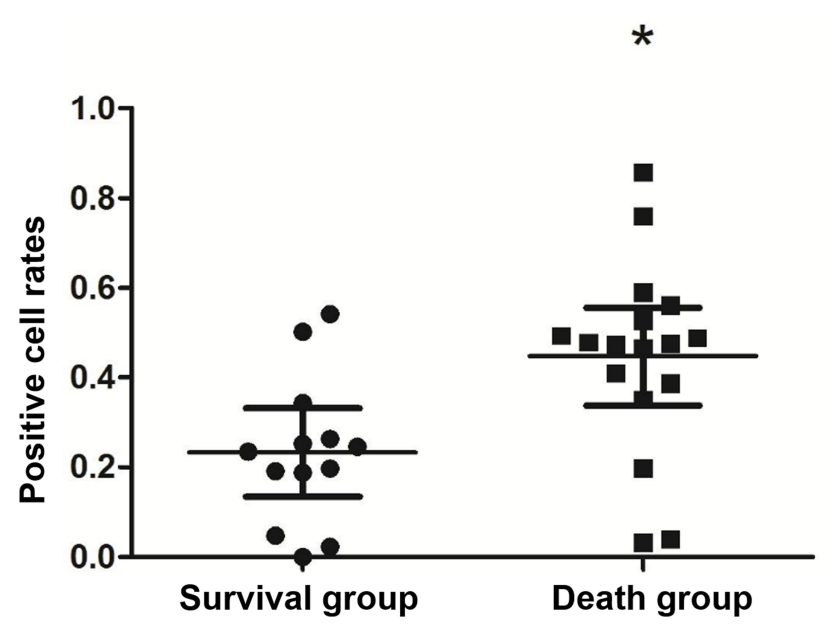

Figure 4 Syncytin-I positive cell rate in 5-year survival group and 5-year death group. The syncytin-I positive cell rate of the carcinoma tissues in the 5-year death group was higher than that in the 5-year survival group. *Represents $\mathrm{P}<0.05$.

\section{Comparison of Total Methylation Ratio and $C_{p}$ G2 Site Methylation Ratio}

The total methylation ratios of NSCLC tissues and paracarcinoma tissues were $77.50 \% \pm 10.19 \%$ and $78.87 \% \pm$ $11.54 \%$, respectively (Figure 6), with no significant difference $(\mathrm{P}>0.05)$. The $\mathrm{CpG} 2$ site methylation ratios of NSCLC tissues and para-carcinoma tissues were $81.91 \% \pm 12.38 \%$ and $94.33 \% \pm 4.93 \%$, respectively, showing a significant difference between the two groups $(\mathrm{P}<0.05)$ (Figure 7, Table 2) according to the non-parametric rank sum test and Mann-Whitney $U$-test of two independent samples.

\section{Discussion}

Lung cancer was particularly prevalent during the twentieth century due to the popularity of tobacco consumption, especially in developed countries where lung cancer ranks at the top of the morbidity and mortality list. The carcinogenesis of lung cancer is a complicated process influenced by environmental, genetic, epigenetic, physical, chemical, immunological, and infectious factors, e.g., the activation of oncogenes and inactivation of suppressor genes. An earlier diagnosis and therapy would lead to a more optimistic prognosis. The 5-year survival ratio of local lung cancer or bronchogenic carcinoma is $54.8 \%$, while this ratio drops sharply to $27.4 \%$ and even $4.2 \%$, respectively, for regional and widespread lung cancers. ${ }^{13}$ Hence, a novel lung cancer biomarker could be essential for developing tools for early diagnosis. Our results suggest that the increased syncytin-1 expression and/or the alteration of DNA methylation in 


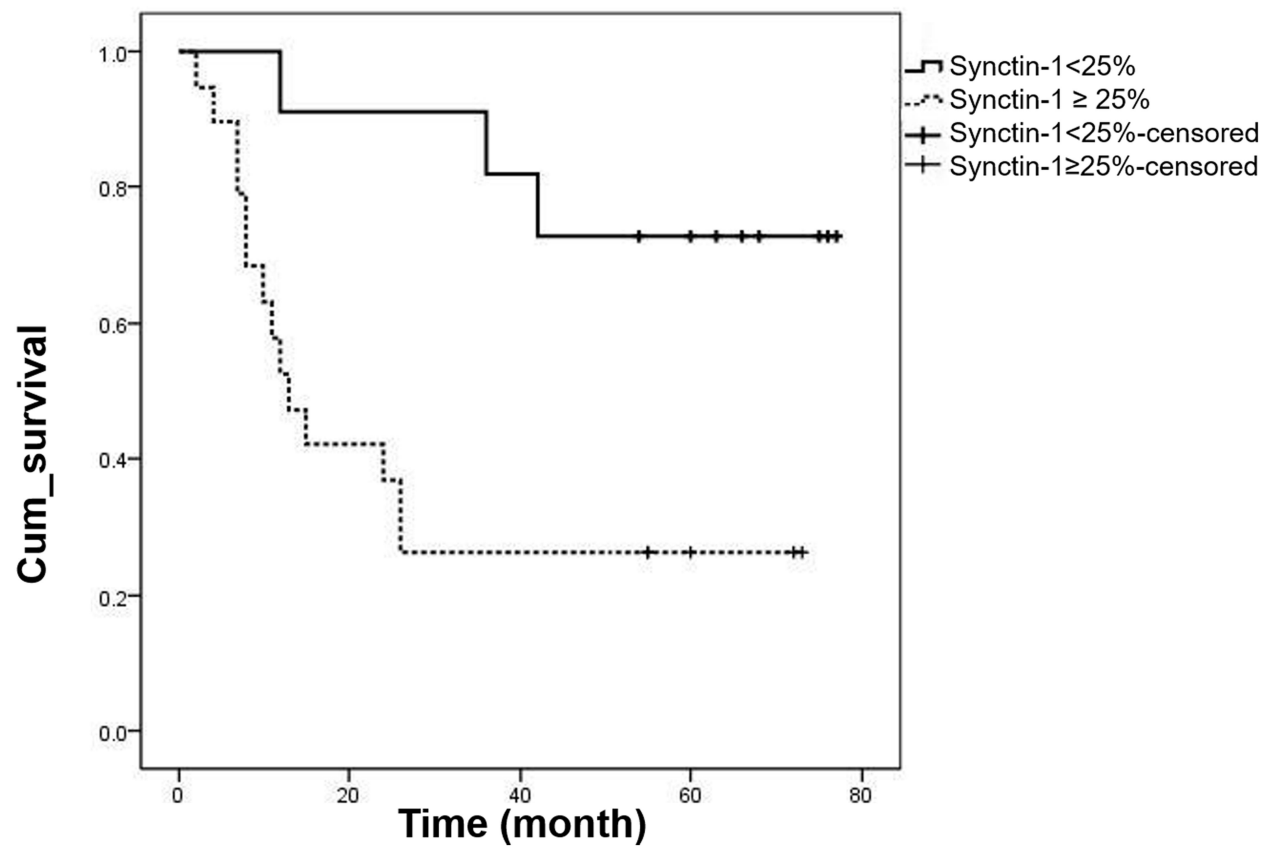

Figure 5 Survival curve of high expression and low expression of Syncytin-I. Kaplan-Meier survival curve analysis showed that the syncytin-I positive cell rate low expression group had a higher recurrence-free survival rate.

the 5'-LTR of $H E R V W$ promoter could be potential biomarkers for the diagnosis of lung cancers.

Larsen et al. ${ }^{16}$ performed a retrospective analysis on 140 colon and rectum cancer cases. It was found that syncytin-1 was commonly expressed in both carcinoma of colon and rectum, and the rectum carcinomas showed high syncytin-1 expression levels as well as low 5-year survival ratios. On the other hand, Larsson et al. ${ }^{10}$ reported that syncytin-1 expression could be an independent positive indicator for breast cancer prognosis since patients with higher syncytin-1 expression in their tumors had a higher non-palindromic survival ratio. In this study, higher expression of syncytin-1 in NSCLC tissue than in

Table I Multivariate Cox Regression Analysis Results of NonSmall Cell Lung Cancer Patients

\begin{tabular}{|c|c|c|c|}
\hline Characteristics & HR & $95 \% \mathrm{Cl}$ & P-value \\
\hline Age, years $(<60 / \geq 60)$ & 0.639 & $0.179-2.273$ & 0.489 \\
\hline Sex (male/female) & 0.678 & $0.176-2.617$ & 0.573 \\
\hline Syncytin-I $(<25 \% / \geq 25 \%)$ & 7.824 & $1.138-53.814$ & $0.037^{*}$ \\
\hline Lymph node metastasis (N0/NI/N2) & 0.449 & $0.160-1.263$ & 0.129 \\
\hline Stage $(I / / / I I I)$ & 8.158 & $2.211-30.093$ & $0.002 *$ \\
\hline Depth of tumor (TI/T2/T3) & 1.258 & $0.506-3.126$ & 0.621 \\
\hline
\end{tabular}

Note: $* \mathrm{P}<0.05$.

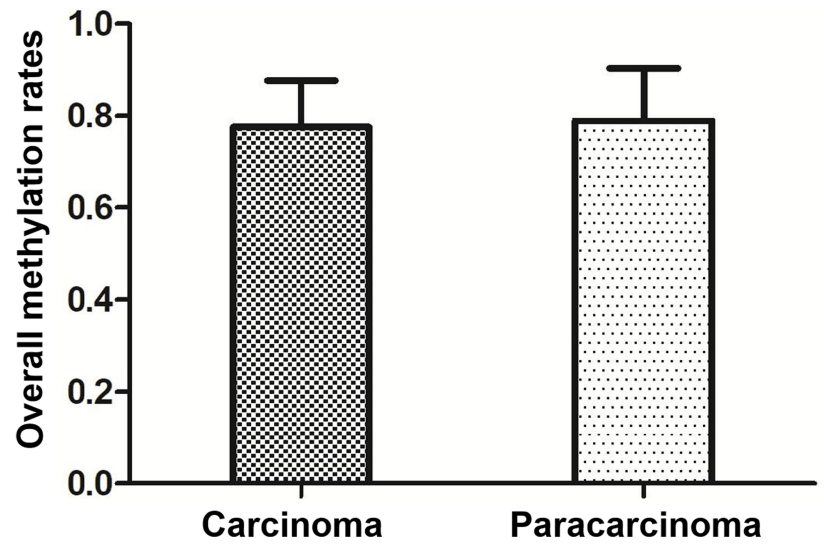

Figure 6 Comparison of overall methylation rates in NSCLC tissues and paracarcinoma tissues. The overall methylation rate of $5^{\prime}$-LTR in the promoter region ofHERVW in NSCLC tissues was lower than that of adjacent tissues.

para-carcinoma tissue as shown by immunohistochemistry is consistent with previous studies in other carcinoma tissues. However, the syncytin-1 expression level was higher in the 5-year death group than the survival group, and the K-M survival curve also demonstrated that the survival ratio in the low-expression group was $27.3 \%$ (3/ 11) with an average survival time of 64 months, while the ratio in the high-expression group was $73.7 \%(14 / 19)$ with an average survival time of only 27 months. This finding suggested that for NSCLC, a high syncytin-1 expression level is associated with a worse prognosis. Among the 


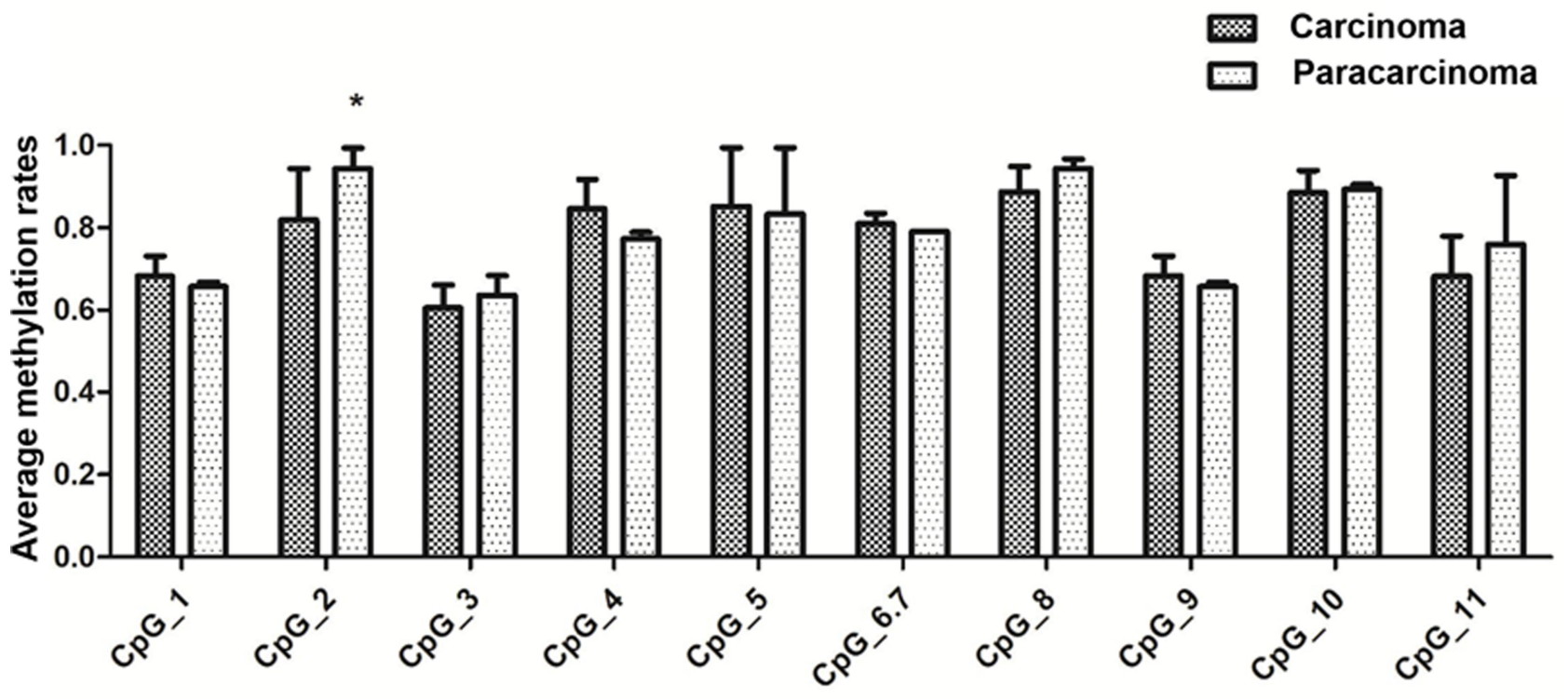

Figure 7 Comparison of average methylation rates of CPG sites in NSCLC tissues and para-carcinoma tissues. The average methylation rate of 10 CPG loci of 5'-LTR in the promoter region of HERVW in carcinoma tissues and para-carcinoma tissues was detected, and the difference in methylation rate of $C_{p}{ }_{2}$ loci was statistically significant. *Represents $\mathrm{P}<0.05$.

factors considered, including age, gender, syncytin-1 positive cell percentage, clinical stage and TNM stage, and clinical stage, syncytin-1 positive cell percentage was found to be the most important factor of NSCLC mortality according to the Cox ratio risk recurrent model. Hence, syncytin-1 could be a novel biomarker for NSCLC diagnosis and prognosis.

Table 2 Comparison of Methylation Rates of CpG Sites in NonSmall Cell Lung Cancer Tissues and Adjacent Tissues

\begin{tabular}{|l|l|l|l|l|}
\hline $\begin{array}{l}\text { Gene } \\
\text { Locus }\end{array}$ & $\begin{array}{l}\text { NSCLC } \\
\text { Tissues } \\
\mathbf{M}(\%)\end{array}$ & $\begin{array}{l}\text { Adjacent } \\
\text { Tissues } \\
\mathbf{M}(\%)\end{array}$ & Z-Vvalue & P-value \\
\hline CPG_I & 68.25 & 65.67 & -0.729 & 0.536 \\
\hline CPG_2 & 81.91 & 94.33 & -2.171 & $0.031 *$ \\
\hline CPG_3 & 60.50 & 63.67 & -0.651 & 0.536 \\
\hline CpG_4 & 84.58 & 77.33 & -1.592 & 0.136 \\
\hline CPG_5 & 85.16 & 83.33 & -0.292 & 0.840 \\
\hline CPG_6.7 & 80.91 & 79.00 & -1.574 & 0.136 \\
\hline CpG_8 & 88.75 & 94.33 & -1.447 & 0.180 \\
\hline CPG_9 & 68.25 & 65.67 & -0.729 & 0.536 \\
\hline CPG_10 & 88.50 & 89.33 & -0.507 & 0.633 \\
\hline CpG_11 & 68.16 & 76.00 & -0.795 & 0.448 \\
\hline
\end{tabular}

Note: $* P<0.05$.

Abbreviation: $\mathrm{M}$, methylation rate.
Syncytin-1 mediates the fusion of chorionic trophoblasts (CT) to form syntrophoblasts (ST), and the reduction in syncytin-1 expression plays an essential role in the pathogenesis of intrauterine growth restriction (IUGR), preeclampsia (PE) and HELLP syndrome. ${ }^{17}$ Besides the function in cell fusion, decreased syncytin-1 expression led to cell apoptosis as observed in preeclampsia. ${ }^{18,19}$ Syncytin-1 was also reported to promote cell proliferation through activation of TGF- $\beta 1$ and TGF- $\beta 3$, to mediate immunosuppression during adaptation to pregnancy. ${ }^{20,21}$ Over $75 \%$ of bladder urothelial carcinoma has up-regulated syncytin-1 expression, which contributes to the proliferation of urothelial cells. ${ }^{22}$ Moreover, in the neuroblastoma cell line, manipulation of syncytin-1 led to abnormal regulation of the cAMP signaling pathway, and toxic excitation of neurons and subsequent neural system diseases. ${ }^{23}$ Thus, non-fusogenic activities of syncytin-1 may be involved in the regulation of a variety of functions in tumor cells. More studies are required to verify if NSCLC could be one of such tumors.

The high expression of syncytin-1 appears to be associated with the low-level methylation of 5'-LTR promoter. Matouskova et al. argue that syncytin-1 expression in trophocytes is triggered by demethylation of the promoter region resulting in proliferation and differentiation of syntrophoblast cells. ${ }^{24}$ Zhuang et al. reported syncytin-1 down-regulation and increased 5'-LTR methylation in the preeclamptic placenta. ${ }^{25}$ Zhou et al. reported that syncytin1 over-expression correlated with a decreased promoter 
methylation level in endometrial cancers. ${ }^{26}$ Benesova et al. compared spermocytoma and nonseminoma and found a low-level methylation of $H E R V W$ promoter in the former and high-level methylation in the latter. ${ }^{27}$ In endometrial cancers, hypomethylated 5'-LTR was associated with upregulated expression of syncytin-1. ${ }^{11}$

At least two components, that is, U3 region of 5'-LTR promoter and its upstream TSE function to regulate the transcription of $H E R V W$ gene owing to the CAAT box and octamer protein binding site of U3 region and $5^{\prime}$-LTR upstream GCMa binding site, which interacts with GCMa transcription factor to increase syncytin-1 expression level in BeWo and JEG3 choriocarcinoma cell lines. ${ }^{21,28,29}$ In this study, $10 \mathrm{CpG}$ sites of $5^{\prime}$-LTR promoter of HERVW gene in NSCLC and para-carcinoma tissues have been investigated (Figure 8). The result demonstrates that HERVW promoter in NSCLC tissues has a relatively lower level of methylation than in para-carcinoma tissues, especially in the $\mathrm{CpG} 2$ site, suggesting a site-specific methylation of HERVW promoter in NSCLC tissue. Our findings are generally consistent with the above observations, all point to an epigenetic regulation of syncytin-1 expression in tumor cells.

Further analysis of the $\mathrm{CpG} 2$ site located 95 nt downstream from the transcriptional start site found it overlaps with an Oct-1 transcription factor binding site. Oct-1 is a DNA-binding transcriptional factor regulating a large number of target genes including housekeeping genes and specific genes involved in immune, endocrine, and neural functions. ${ }^{30}$ High levels of Oct-1 expression have been found in somatic stem cells and cancer stem cells, and gastric, prostatic, cervical, and esophageal cancers. ${ }^{31}$ $H E R V W$ promoter demethylation may result in an increased binding by transcriptional factors such as Oct1 , to induce the expression of syncytin-1 protein. Thus, the CpG2 site could be a key epigenetic component mediating epigenetic and/or non-epigenetic regulation mechanisms of syncytin-1 expression under different sorts of pathological conditions.

The study had several limitations. Firstly, only one methylation site was found to show significant differences, which led to insufficient evidence on the relationship between methylation and protein expression, which may be related to the small number of samples or stale samples. Therefore, in the subsequent study, we will collect fresh tissue samples to carry out a multi-center joint study.

In this study, an over-expression of syncytin-1 was found in NSCLC tissues compared with adjacent tissues. The overexpression of syncytin-1 was associated with a worse prognosis of patient survival. A low-methylation level in the 5'-LTR promoter region of the HERVW gene, especially a specific $\mathrm{CpG}$ site overlapping with an oct-1 binding site was identified as a key element for the regulation of syncytin-1 expression. Although the precise molecular mechanism remains elusive, the observations suggest that syncytin-1 could be a novel biomarker for lung cancer diagnosis/prognosis, and further studies along this line
5'LTR

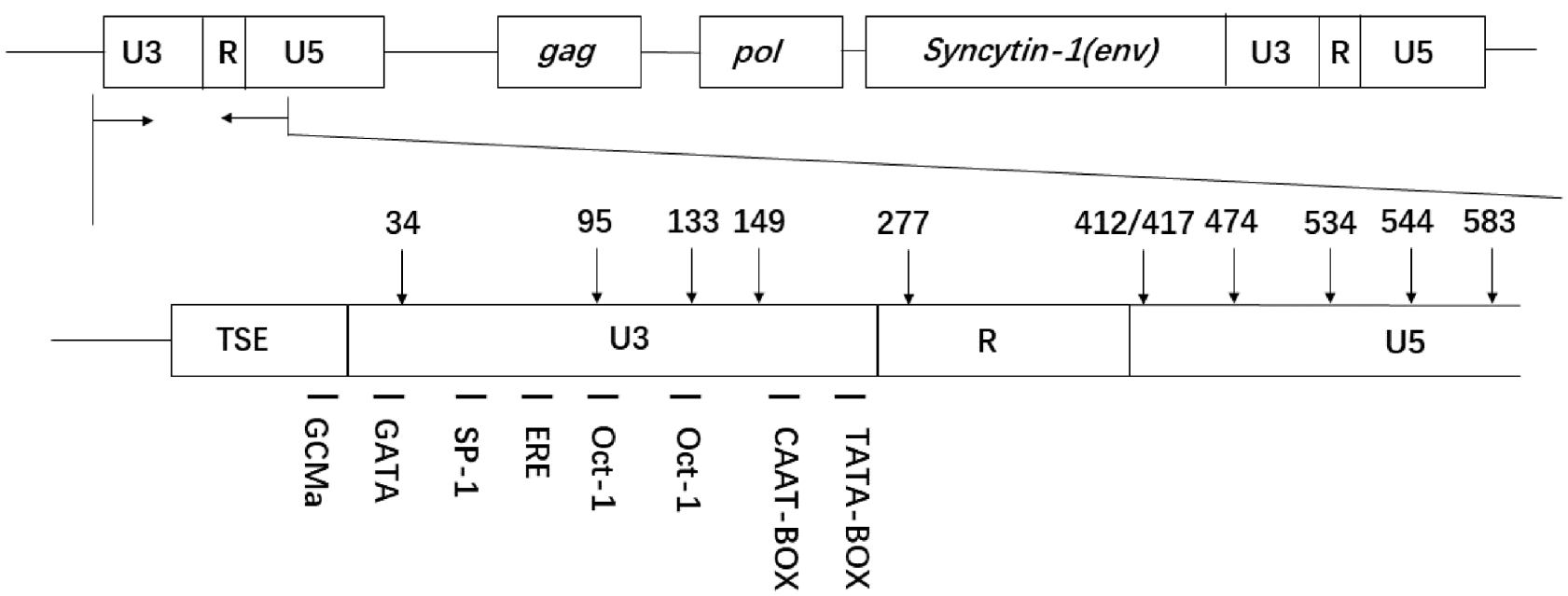

Figure 8 Schematic map of theHERWW gene. The downward arrow and number indicate the $10 \mathrm{CPG}$ sites and position in the methylated fragment, respectively. The TSE is the trophoblast-specific enhancer region located directly upstream of the 5'LTR U3 region. In addition, important cis-acting elements are highlighted below the methylated fragment. 
may be useful for improving the management of lung cancers.

\section{Abbreviations}

NSCLC, non-small cell lung cancer; MF, muscarinic fungoides; CT, chorionic trophoblast; ST, syntrophoblast; IUGR, intrauterine growth restriction; PE, preeclampsia.

\section{Ethical Approval}

Ethical approval for this project was obtained from the Ethics Committee of Qilu Hospital, Shandong University. All procedures performed in studies involving human participants were in accordance with the ethical standards of the institutional and/or national research committee and with the 1964 Helsinki declaration and its later amendments or comparable ethical standards.

\section{Informed Consent}

Written informed consent was obtained from all individual participants included in the study.

\section{Acknowledgments}

This study was supported by grants from Shandong Provincial Nature Science Foundation (No. ZR2015CM030), Shandong Key Research and Development Program (No. 2016GSF201169).

\section{Disclosure}

The authors declare that they have no conflicts of interest.

\section{References}

1. Balgkouranidou I, Liloglou T, Lianidou ES. Lung cancer epigenetics: emerging biomarkers. Biomark Med. 2013;7(1):49-58. doi:10.2217/ bmm.12.111

2. Herbst RS, Heymach JV, Lippman SM. Lung cancer. $N$ Engl J Med. 2008;359(13):1367-1380. doi:10.1056/NEJMra0802714

3. Siegel RL, Miller KD, Jemal A. Cancer statistics, 2020. CA Cancer $J$ Clin. 2020;70(1):7-30. doi:10.3322/caac.21590

4. Molina R, Filella X, Auge JM, et al. Tumor markers (CEA, CA 125, CYFRA 21-1, SCC and NSE) in patients with non-small cell lung cancer as an aid in histological diagnosis and prognosis. Comparison with the main clinical and pathological prognostic factors. Tumour Biol. 2003;24(4):209-218. doi:10.1159/000074432

5. Nanavaty P, Alvarez MS, Alberts WM. Lung cancer screening: advantages, controversies, and applications. Cancer Control. 2014;21 (1):9-14. doi:10.1177/107327481402100102

6. Blond JL, Besème F, Duret L, et al. Molecular characterization and placental expression of HERV-W, a new human endogenous retrovirus family. $J$ Virol. 1999;73(2):1175-1185. doi:10.1128/JVI.73.2.1175-11 85.1999

7. Voisset C, Bouton O, Bedin F, et al. Chromosomal distribution and coding capacity of the human endogenous retrovirus HERV-W family. Aids Res Hum Retroviruses. 2000;16(8):731-740. doi:10.1089/08892 2200308738
8. Maliniemi P, Vincendeau M, Mayer J, et al. Expression of human endogenous retrovirus-w including syncytin-1 in cutaneous T-cell lymphoma. PLoS One. 2013;8(10):e76281. doi:10.1371/journal. pone.0076281

9. Sun Y, Zhu H, Song J, et al. Upregulation of leukocytic syncytin-1 in acute myeloid leukemia patients. Med Sci Monit. 2016;22:23 92-2403. doi:10.12659/MSM.899303

10. Larsson LI, Holck S, Christensen IJ. Prognostic role of syncytin expression in breast cancer. Hum Pathol. 2007;38(5):726-731. doi:10.1016/j.humpath.2006.10.018

11. Strissel PL, Ruebner M, Thiel F, et al. Reactivation of codogenic endogenous retroviral (ERV) envelope genes in human endometrial carcinoma and prestages: emergence of new molecular targets. Oncotarget. 2012;3(10):1204. doi:10.18632/oncotarget.679

12. Brzeziańska E, Dutkowska A, Antczak A. The significance of epigenetic alterations in lung carcinogenesis. Mol Biol Rep. 2013;40 (1):309-325. doi:10.1007/s11033-012-2063-4

13. Ansari J, Shackelford RE, Elosta H. Epigenetics in non-small cell lung cancer: from basics to therapeutics. Transl Lung Cancer Res. 2016;5(2):155. doi:10.21037/tlcr.2016.02.02

14. Jones PA, Baylin SB. The epigenomics of cancer. Cell. 2007;128 (4):683-692. doi:10.1016/j.cell.2007.01.029

15. Bolze PA, Mommert M, Mallet F. Contribution of syncytins and other endogenous retroviral envelopes to human placenta pathologies. Prog Mol Biol Transl Sci. 2017;145:111-162.

16. Larsen JM, Christensen IJ, Nielsen HJ, et al. Syncytin immunoreactivity in colorectal cancer: potential prognostic impact. Cancer Lett. 2009;280(1):44-49. doi:10.1016/j.canlet.2009.02.008

17. Ruebner M, Strissel PL, Ekici AB, et al. Reduced syncytin-1 expression levels in placental syndromes correlates with epigenetic hypermethylation of the ERVW-1 promoter region. PLoS One. 2013;8(2): e56145. doi:10.1371/journal.pone.0056145

18. Huang Q, Chen H, Wang F, et al. Reduced syncytin-1 expression in choriocarcinoma BeWo cells activates the calpain1-AIF-mediated apoptosis, implication for preeclampsia. Cell Mol Life Sci. 2014;71 (16):3151-3164. doi:10.1007/s00018-013-1533-8

19. Langbein M, Strick R, Strissel PL, et al. Impaired cytotrophoblast cell-cell fusion is associated with reduced syncytin and increased apoptosis in patients with placental dysfunction. Mol Reprod Dev. 2008;75(1):175-183. doi:10.1002/mrd.20729

20. Lu Q, Li J, Senkowski C, et al. Promoter hypermethylation and decreased expression of syncytin-1 in pancreatic adenocarcinomas. PLoS One. 2015;10(7):e0134412. doi:10.1371/journal.pone.0134412

21. Yu C, Shen K, Lin M, et al. GCMa regulates the syncytin-mediated trophoblastic fusion. J Biol Chem. 2002;277(51):50062-50068. doi:10.1074/jbc.M209316200

22. Yu H, Liu T, Zhao Z, et al. Mutations in 3|[prime]|-long terminal repeat of HERV-W family in chromosome 7 upregulate syncytin-1 expression in urothelial cell carcinoma of the bladder through interacting with c-Myb. Oncogene. 2013;33(30):3947-3958. doi:10.1038/ onc. 2013.366

23. Li S, Liu ZC, Yin SJ, et al. Human endogenous retrovirus $\mathrm{W}$ family envelope gene activates the small conductance $\mathrm{Ca} 2$ +-activated $\mathrm{K}+$ channel in human neuroblastoma cells through CREB. Neuroscience. 2013;247(13):164-174. doi:10.1016/j. neuroscience.2013.05.033

24. Matousková M, Blazková J, Pajer P, Pavlícek A, Hejnar J. CpG methylation suppresses transcriptional activity of human syncytin-1 in non-placental tissues. Exp Cell Res. 2006;312(7):1011-1020.

25. Zhuang XW, Li J, Brost BC, et al. Decreased expression and altered methylation of syncytin-1 gene in human placentas associated with preeclampsia. Curr Pharm Des. 2014;20(11):1796-1802. doi:10.21 74/13816128113199990541

26. Zhou H, Li J, Podratz KC, et al. Hypomethylation and activation of syncytin-1 gene in endometriotic tissue. Curr Pharm Des. 2014;20 (11):1786. doi:10.2174/13816128113199990540 
27. Benešová $\mathrm{M}$, Trejbalová $\mathrm{K}$, Kovářová $\mathrm{D}$, et al. DNA hypomethylation and aberrant expression of the human endogenous retrovirus ERVWE1/syncytin-1 in seminomas. Retrovirology. 2017;14(1):20. doi:10.1186/s12977-017-0342-9

28. Cheng YH, Richardson BD, Hubert MA, Handwerger S. Isolation and characterization of the human syncytin gene promoter. Biol Reprod. 2004;70(3):694-701. doi:10.1095/biolreprod.103.023473

29. Gimenez J, Montgiraud C, Oriol G, et al. Comparative methylation of ERVWE1/syncytin-1 and other human endogenous retrovirus LTRs in placenta tissues. DNA Res. 2009;16(4):195-211. doi:10.1093/ dnares/dsp011
30. Pankratova EV, Stepchenko AG, Krylova ID, Portseva TN, Georgieva SG. The regulatory interplay between Oct- 1isoforms contributes to hematopoiesis and the isoforms imbalance correlates with a malignant transformation of B cells. Oncotarget. 2018;9 (52):29892-29905. doi:10.18632/oncotarget.25648

31. Pankratova EV, Stepchenko AG, Portseva T, Mogila VA, Georgieva SG. Different N-terminal isoforms of Oct- 1 control expression of distinct sets of genes and their high levels in namalwa burkitt's lymphoma cells affect a wide range of cellular processes. Nucleic Acids Res. 2016;44(19):9218-9230.

\section{Publish your work in this journal}

The International Journal of General Medicine is an international, peer-reviewed open-access journal that focuses on general and internal medicine, pathogenesis, epidemiology, diagnosis, monitoring and treatment protocols. The journal is characterized by the rapid reporting of reviews, original research and clinical studies across all disease areas. The manuscript management system is completely online and includes a very quick and fair peer-review system, which is all easy to use. Visit http://www.dovepress.com/ testimonials.php to read real quotes from published authors.

Submit your manuscript here: https://www.dovepress.com/international-journal-of-general-medicine-journal 